\title{
Population genetics of neotropical trees focus issue
}

\section{Heredity (2005) 95, 243-245. doi:10.1038/sj.hdy.6800755}

Few can fail to be impressed by the amazing biodiversity and complexity of tropical forest ecosystems. Decades of work from biologists in a range of disciplines, including ecological, reproductive, behavioural, dispersal and evolutionary biology, have provided insight into the processes and functioning of these systems. Perhaps the most thoroughly investigated tropical ecosystems are the forests of the neotropics, and in particular the components of biodiversity and ecological interactions of their tree species (eg pollen and seed dispersal mechanisms and vectors). Over the last decade, the application of molecular markers in particular has led to a dramatic improvement in our knowledge of the historical and contemporary processes that contribute to the level, structure and functioning of biodiversity in this globally important bioregion.

In many cases, the scientific questions posed within tropical forests are unique to those ecosystems. For example, what has driven the large-scale diversification within the tropics, and how do so many low-density species retain genetic connectivity? What is the nature of the mutualistic relationships between plants and their pollinators and seed dispersers, how did these relationships evolve, and what are the resultant gene dispersal implications of these mechanisms? Finally, with the widespread and increasing rates of deforestation and logging within neotropical countries, new concerns are arising. For example, how do species cope with life in small isolated remnants that result from loss of habitat?

Many researchers and scientific groups from around the globe have contributed to the development of knowledge and scientific study in the neotropics. In addition to scientists working out of national or region research institutes and universities in Latin America (from Mexico to Argentina), many scientists from North America and Europe have been fortunate to receive funding to study some of these amazing systems. Lasting research infrastructures have also been put into place in many regions, by either setting up permanent sample plots or providing roads, buildings and even aerial access facilities to allow better study of forests and canopy interactions. Such infrastructure has contributed significantly to the ability to undertake long-term studies in what can be at times a challenging environment.

In this special focus issue of Heredity, we present a group of six papers focusing on aspects of neotropical population genetics, and which represent a product of this scientific investment. Four of the six assembled papers are the culmination of collaborative work between Latin American and European scientists (from Belgium, Brazil, Costa Rica, France, French Guyane, Germany, Panama, United Kingdom) and funded by a series of EU projects in conjunction with national partner grants (through the INCO-DEV programmes in frameworks 3, 4 and 5, and including the project GENEO-
TROPECO). The other two papers are collaborative outputs of researchers based either in Panama, French Guyane and USA, or Mexico and USA. These six papers take a variety of forms, including two review papers, two papers reporting methodological advancements and two papers reporting primary research results; they represent a range of issues currently being tackled, debated and developed in neotropical systems.

The reviews represent summaries of the contemporary neotropical literature in two important areas. The review by Ward et al (2005) offers insight into some of the key mating system and gene flow mechanisms that individual species adopt to maintain genetic connectivity in the notoriously low-density conditions of many rainforests. Taking its starting point from earlier reviews by Murawski (1995) and Loveless (2002), this review examines levels of selfing and gene flow across a range of species case studies. In contrast to very early presumptions that most species must be selfing to survive such low-density conditions, a survey of the contemporary molecular marker literature indicates that most neotropical species are highly outcrossed. Indeed, many have a range of very sophisticated pollination syndromes to overcome their low-density lifestyles. Mixed mating systems are still found in a small proportion of species, but these have so far been confined to a single family, the Malvaceae. Future recommendations of this review include examination of inbreeding depression on observed outcrossing rates, quantification of pollen dispersal for more species, including sampling wider ecological classes, and examination of variation in mating systems due to individual, seasonal, population, ecological and landscape differences.

With deforestation and logging rates in the neotropics being some of the highest in the world, concern over the state of the genetic resources of exploited and forest dependent species is paramount. The review of Lowe et al (2005) provides a picture of the level of impact these processes are having on the component genetic diversity of neotropical trees. This review takes its starting point from an earlier synthesis by Young et al (1996), which covered mostly temperate case studies but which also identified the lack of research in tropical systems. Since this earlier review, many new studies have been published, and Lowe et al highlight some of these recent findings. Most notably, simulation and empirical studies appear to indicate that genetic diversity and differentiation estimates are relatively robust to reductions in population size from habitat fragmentation and logging. Even in heavily exploited populations, the longevity of trees and overlapping generations appear to buffer diversity loss. However, degraded populations suffer significantly from increased inbreeding, reduced reproductive output and decreased fitness (which appears to be due to both inbreeding and ecological effects of tree isolation). Unexpectedly, some studies highlight that extensive networks of gene flow, established at medium spatial scales in fragmented landscapes (up to $10 \mathrm{~km}$ ), 
can mitigate against genetic diversity loss and assist long-term population viability. Suggested future research priorities include undertaking integrated studies on a range of species in the same landscapes, better documentation of the extent and duration of impact, and, most importantly, combining neutral marker, pollination dynamics, ecological consequences and progeny fitness assessment within single studies.

The two methodologically orientated papers advise on developments in statistical and sampling techniques relevant to surveys of the level and structure of genetic diversity within neotropical tree populations. The first paper, by Kremer et al (2005), examines how genetic diversity estimates can best be made from AFLP loci, a molecular marker type that has been widely applied to tropical species due to a lack of previous genomic investigation. Building on earlier statistical developments (Lynch and Milligan, 1994; Zhivotovsky, 1999; Krauss, 2000), this paper shows that, while the fixation index of populations is not readily accessible from dominant markers, variations in this parameter have little impact on multilocus estimates of genetic diversity. The robustness of genetic diversity estimates is due to compensatory negative and positive biases at individual AFLP loci that exhibit contrasting frequency profiles of null alleles. The paper gives practical recommendations for implementing this analytical method that should allow for more accurate estimation of genetic diversity in tropical trees.

Surveys of fine-scale genetic structure within tree populations are increasingly being used to infer smallscale pollen and seed dispersal distances and processes (eg Hardy and Vekemans, 1999; Fenster et al, 2003; Vekemans and Hardy, 2004). DNA samples can now easily be obtained from trees at ground level (by taking cambium samples; Colpaert et al, 2005); so gene flow can be studied without needing to sample open-pollinated seed from tropical canopies. The second methodological paper, by Cavers et al (2005), examines the best sampling strategies, for detecting spatial genetic structure within populations, both in terms of number of individuals and number of loci. Simulated and experimental data for both codominant and dominant markers are scrutinized for suitability of sample size. Earlier work recommends that 300-400 individuals be sampled (Geburek and TrippKnowles, 1994). In contrast, Cavers et al (2005) find that maximum efficiency (a tradeoff between statistical robustness and sampling effort) is achieved by taking between 100 and 200 individuals for 10-5 microsatellite loci, respectively, or between 150 and 200 individuals for 100 AFLP loci. They apply these guidelines to a series of neotropical case studies and offer a set of practical guidelines for surveys of spatial genetic structure.

Finally, two primary research papers describe analyses of spatial genetic structure of key components of Latin American forests. Luna et al (2005) provide one of the first papers to examine spatial genetic structure within palm species. They analyse two species with contrasting life history characteristics, Chamaedorea tepejilote and Chamaedorea elatior, from a single sympatric population in Mexico. Both palms exhibited spatial genetic structure, consistent with highly dispersed species. In addition, a range of factors were found to affect standing spatial genetic structure, including seed dispersal, mortality among life stages, overlapping generations and contrasting traits of mating and reproduction.
A second paper, by Hardesty et al (2005), reports an analysis of spatial genetic structure of Symphonia globifera, sampled from the Smithsonian Tropical Research Institute's permanent plot on Barro Colorado Island, Panama. In contrast to previous studies of this vertebrate-dispersed, insect-pollinated tree, low levels of spatial genetic structure were identified using both microsatellite and AFLP markers. This discrepancy is best explained by overlapping seed shadows, postrecruitment mortality, and pollen and seed dispersal over the scale of the 50 hectare sample plot. This group of papers demonstrates the rapid expansion of both the methodological and empirical base of population genetic research in neotropical systems.

A Lowe
School of Integrative Biology, University of Queensland,
Brisbane, Queensland 4072, Australia
E-mail: a.lowe@uq.edu.au

\section{References}

Cavers S, Degen B, Caron H, Hardy O, Lemes M, Gribel R et al (2005). Optimal sampling strategy for estimation of spatial genetic structure in tree populations. Heredity 95: 281-289.

Colpaert N, Cavers S, Bandou E, Caron H, Gheysen G, Lowe AJ (2005). Sampling tissue for DNA analysis of trees: trunk cambium as an alternative to canopy leaves. Silvae Genet, in press.

Fenster CB, Vekemans X, Hardy OJ (2003). Quantifying gene flow from spatial genetic structure data in a metapopulation of Chamaecrista fasciculata (Leguminosae). Evolution 57: 995-1007.

Geburek T, Tripp-Knowles P (1994). Spatial stand structure of sugar maple (Acer saccharum Marsh) in Ontario, Canada. Phyton-Ann REI Bot 34: 267-278.

Hardesty BD, Dick CW, Kremer A, Hubbell S, Bermingham E (2005). Spatial genetic structure of Simarouba amara Aubl. (Simaroubaceae), a dioecious, animal-dispersed Neotropical tree, on Barro Colorado Island, Panama. Heredity 95: 290-297.

Hardy OJ, Vekemans X (1999). Isolation by distance in a continuous population: reconciliation between spatial autocorrelation analysis and population genetics models. Heredity 83: $145-154$

Krauss SL (2000). Accurate gene diversity estimates from amplified fragment length polymorphism (AFLP) markers. Mol Ecol 9: 1241-1245.

Kremer A, Caron H, Cavers S, Colpaert N, Gheysen L, Gribel R et al (2005). Monitoring genetic diversity in tropical trees with multilocus dominant markers. Heredity 95: 274-280.

Loveless MD (2002). Genetic diversity and differentiation in tropical trees. In: Degen B, Loveless MD, Kremer A (eds) Modelling and Experimental Research on Genetic Processes in Tropical and Temperate Forest, Embrapa. pp 3-30.

Lowe AJ, Boshier D, Ward M, Bacles CFE, Navarro C (2005). Genetic resource impacts of habitat loss and degradation; reconciling empirical evidence and predicted theory for neotropical trees. Heredity 95: 255-273.

Luna R, Epperson BK, Oyama K (2005). Spatial genetic structure of two sympatric neotropical palms with contrasting life histories. Heredity 95: 298-305.

Lynch M, Milligan BG (1994). Analysis of population genetic structure with RAPD markers. Mol Ecol 3: 91-99.

Murawski DA (1995). Reproductive biology and genetics of tropical trees from a canopy perspective. In: Loma M, Nadkwarm N (eds) Forest Canopies. Academic Press: New York. pp 457-491. 
Vekemans X, Hardy OJ (2004). New insights from fine-scale spatial genetic structure analyses in plant populations. Mol Ecol 13: 921-935.

Ward M, Dick CW, Gribel R, Lemes M, Caron H, Lowe AJ (2005). To self, or not to self... A review of outcrossing and pollen-mediated gene flow in neotropical trees. Heredity 95: 246-254.
Young A, Boyle T, Brown AHD (1996). The population genetic consequences of habitat fragmentation for plants. Trends Eco Evol 11: 413-418.

Zhivotovsky LA (1999). Estimating population structure in diploids with multilocus dominant DNA markers. Mol Ecol 8: 907-913. 